\title{
De la membrane au noyau, un couplage direct entre des récepteurs de cytokines et la machinerie transcriptionnelle
}

En 1992, des progrès considérables ont été réalisés dans la compréhension des mécanismes de transmission des signaux extracellulaires aux machineries intracellulaires, notamment à la machinerie transcriptionnelle. Les deux progrès les plus significatifs concernent l'intervention des petites protéines $G$ de la famille Ras dans la transmission des signaux passant par des récepteurs de cytokines et de facteurs de croissance, dotés ou non d'une activité de tyrosine kinase $\left(\mathrm{m} / \mathrm{s} n^{\circ} 5\right.$, vol. $8, p .471 \mathrm{et}$ $n^{\circ} 10$, vol. $\left.8, p .1095\right)$. Un autre coup de tonnerre fut la démonstration qu'un chemin beaucoup plus direct pouvait être emprunté entre la membrane et le noyau, illustré par le mode d'action des interférons $\alpha$, $\beta$ et $\gamma\left(\mathrm{m} / \mathrm{s} n^{\circ} 8\right.$, vol. $8, p$. 838). Dans ce dernier cas, la liaison des interférons à leurs récepteurs aboutit, par l'intermédiaire de tyrosine kinases cytosoliques, à la phosphorylation de facteurs de transcription qui, ainsi activés, pénètrent dans le noyau, se fixent à leur séquencecible et stimulent la transcription des gènes répondant aux interférons. En 1993, les différentes étapes du signal passant par Ras ont été mieux décrites, les différents partenaires agissant entre le récepteur et la machinerie transcriptionnelle étant maintenant bien caractérisés (m/s $n^{\circ} 10$, vol.9, p 1126). Cependant, c'est principalement le domaine de l'activation directe de facteurs transcriptionnels par des tyrosine kinases qui a explosé, tout spécialement durant le quatrième trimestre de l'année 1993 et au début de 1994. Les résultats obtenus l'ont été grâce à l'utilisation ingénieuse de méthodes de génétique somatique (c'est-à-dire à l'emploi de lignées mutantes de cellules somatiques en culture) et de biochimie.

La transmission du signal des interférons nécessite l'intervention de deux tyrosine kinases

Une équipe dirigée par IM Kerr, à Londres (GB) a utilisé, pour élucider les intermédiaires de la transmission du signal de l'interféron, le type de stratégie qui lui avait permis de démontrer l'intervention de la tyrosine kinase Tyk2 dans les effets biologiques de l'interféron $\alpha(\mathrm{m} / \mathrm{s}$ $n^{\circ} 8$, vol. 8, p. 838), l'emploi de lignées de cellules mutantes ne répondant plus aux interférons. Une lignée incapable de répondre aux interférons $\alpha / \beta$ et $\gamma$ s'est révélée être déficiente en la tyrosine kinase Jakl [1], alors qu'une lignée déficiente pour la réponse à l'interféron $\gamma$ mais normalement stimulée par l'interféron $\alpha$ était déficiente en la tyrosine kinase Jak2 [2]. La transmission du signal provoqué par l'interféron $\alpha$ nécessitant aussi la présence de la tyrosine kinase Tyk2, ces résultats indiquent que l'effet des interférons requiert la présence de deux tyrosine kinases cytoplasmiques, Jak 1 et TyK2 pour l'interféron $\alpha$, Jakl et Jak2 pour l'interféron $\gamma$. Une seule de ces tyrosine kinases est incapable de permettre la transmission du signal. Jakl est commune aux voies de transmission des signaux des interférons $\alpha / \beta$ et $\gamma$. La nécessité d'une coopération entre deux tyrosine kinases différentes dans la trans- mission de ces signaux pourrait être expliquée par plusieurs phénomènes. Les tyrosine kinases ont besoin, pour être activées, d'être ellesmêmes phosphorylées sur des résidus tyrosine. Il se pourrait que cette phosphorylation ne fût pas une auto-phosphorylation, mais le résultat de l'action l'une sur l'autre des deux tyrosine kinases de chaque couple. Par ailleurs, il semble que la présence des deux tyrosine kinases soit indispensable à l'édification d'un récepteur fonctionnel pour les interférons. En présence d'une seule d'entre elles, le signal pourrait être interrompu à l'origine, au niveau d'un complexe-récepteur incomplet.

Les protéine kinases de la famille Jak/Tyk induisent la phosphorylation sur des tyrosines de facteurs de transcription Stat

Nous avons déjà vu que la stimulation par l'interféron $\alpha$ ou $\beta$ entraînait la phosphorylation sur des résidus tyrosine des différentes sousunités du facteur ISGF3 (interferonstimulated gene factor 3), p113 et $\mathrm{p} 91 / \mathrm{p} 84\left(\mathrm{~m} / \mathrm{s} n^{\circ} 8\right.$, vol. 8, p. 838). Les protéines p84 et p91 sont issues de l'épissage différentiel du transcrit d'un même gène ; il manque à p84 38 acides aminés carboxy-terminaux qui pourraient intervenir dans la stimulation transcriptionnelle. Ces facteurs phosphorylés migrent dans le noyau où ils s'associent à p48 pour former un complexe d'activation transcriptionnelle au niveau de l'élément d'ADN ISRE (interferonstimulated response element). Le traitement par l'interféron $\gamma$ n'entraîne la 
phosphorylation que de p9l qui se fixe à l'élément d'ADN GAS $(\gamma$ interferon-activated sequence), situé en amont des gènes stimulés par l'interféron $\gamma$. L'équipe de James Darnell propose de dénommer ces protéines p91 et pl13 des Stat, pour signal transducers and activators of transcription [3]. Jakl et Jak2, préalablement activés à la suite de la liaison de l'interféron $\gamma$ à son récepteur, sont toutes deux indispensables à la phosphorylation de Stat9l sur la tyrosine 701. Symétriquement, la phosphorylation de Statl13 requiert la présence, à la fois, de Jakl et de Tyk2 $[3,4]$
Des voies communes de transmission des signaux existent pour les interférons et d'autres cytokines ou facteurs de croissance

La protéine kinase Jak2, indispensable à la transmission du signal de l'interféron $\gamma$, est également impliquée dans la transmission des signaux de l'érythropoïétine [5], de l'interleukine 3 [6], et de l'hormone de croissance [7]. Si la règle présidant à la transmission des signaux des interférons vaut également pour ces cytokines, Jak2 doit coopérer avec une autre tyrosine kinase qui n'a pas encore été identifiée. De même, les facteurs de type Stat, impliqués dans ces voies de transmission du signal, ne sont pas identifiés; les protéines Stat9l et Stat113 ne sont pas phosphorylées lors du traitement des cellules par l'érythropoïétine, l'interleukine-3 ou l'hormone de croissance.

En revanche, Stat9l est l'un des facteurs transcriptionnels activés lors de la stimulation des cellules par EGF et PDGF (epidermal growth factor et platelet derived growth factor). Ces facteurs stimulent, notamment, la transcription du gène $c$-fos en agissant au niveau d'un élément d'ADN SIE (Sisinducible element) localisé une tren-

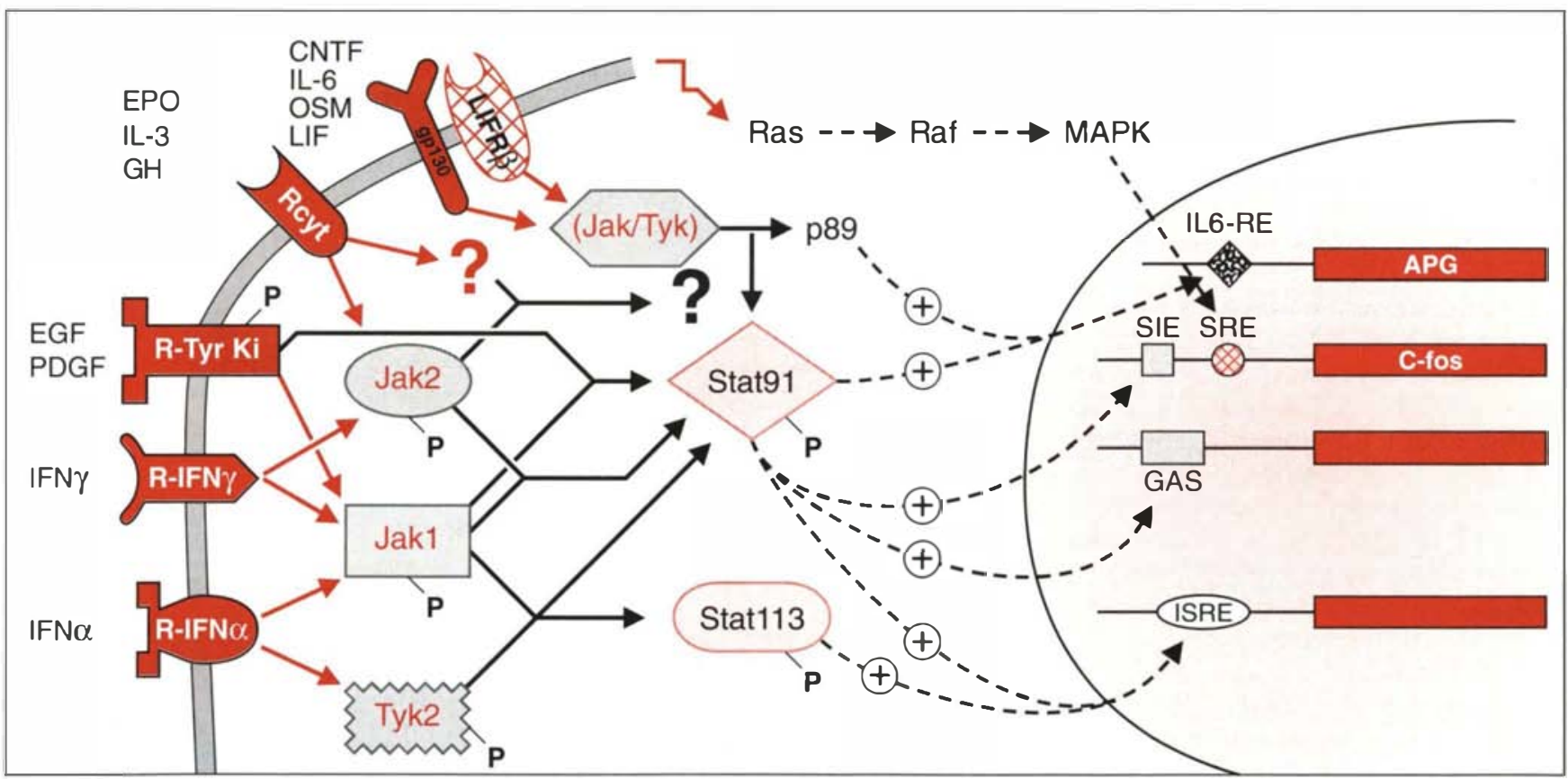

Figure 1. Schéma de la transmission des signaux des interférons, de facteurs de croissance et de cytokines. En rouge, les tyrosine kinases cytoplasmiques impliquées dans la transmission du signal passant par différents récepteurs. En traits noirs continus, les facteurs de transcription cytoplasmiques phosphorylés par l'action combinée de deux de ces tyrosine kinases. Dans le cas du récepteur de l'EGF, la coopération se fait probablement entre Jak1 et l'activité tyrosine kinasique intrinsèque du récepteur. Dans le cas des récepteurs $(R$-cyt pour récepteurs de cytokines) de l'érythropoiétine (EPO), de I'hormone de croissance (GH) et de l'interleukine-3 (IL3), les partenaires éventuels de Jak2 et leurs cibles sont inconnus. Les récepteurs pour les facteurs de la famille CNTF (CNTF, IL6, oncostatine $M$ et LIF) ont en commun la gp130 et, pour CNTF, OSM et LIF, la sous-unité LIFRB. Ces sousunités de récepteurs interagissent avec diverses tyrosine kinases de la famille Jak/Tyr. Les lignes discontinues indiquent les effets des facteurs Stat (signal transducers and activators of transcription) sur différentes cibles d'ADN: IL6-RE (IL6 response elements), SIE (Sis-inducible element), GAS (gamma interferon-activated sequence) et ISRE (interferon-stimulated response element). Les lettres $P$ indiquent la phosphorylation de résidus tyrosine, notamment la tyrosine 701 dans le cas de Stat91. Le gène c-Fos possède également l'élément SRE (serum response element) sur lequel se fixe le complexe p67SRF et p62 TCF. p62TCF est un facteur de transcription de la famille Ets, dénommé Elk1, qui est activé par phosphorylation catalysée par les MAP kinases. Par conséquent, l'élément SRE est la cible de la voie de transmission du signal passant par des récepteurs de facteurs de croissance ou de cytokines, Ras, Raf et les MAP kinases. APG: acute phase gene, répondant à IL6. 
taine de bases en amont de l'élément SRE (serum response element). SIE fixe le facteur SIF (Sis-inducible factor). La séquence de l'élément SIE est très voisine de celle de l'élément GAS conférant la réponse à l'interféron $\gamma$. De plus, parmi les protéines capables de se fixer à SIE, l'une d'entre elles est incontestablement Stat9l [8-12]. Stat9l pourrait également être impliquée dans la transmission du signal d'autres cytokines, notamment de l'interleukine-6 au niveau des cellules hépatiques [9], et de l'interleukine-10 [13]. En réalité, différentes cytokines semblent activer différentes combinaisons de facteurs de transcription ayant des préférences de fixation pour des éléments d'ADN de la famille GAS/SIE $[8,11,13]$, permettant d'expliquer, tout à la fois, le recouvrement et la spécificité des actions des différentes cytokines et des facteurs de croissance.

\section{La triple combinatoire des récep teurs de cytokines, des tyrosine kina- ses et des facteurs de transcription}

La transmission des signaux issus des facteurs CNTF (ciliary neurotrophic factor), OSM (oncostatin M), I.IF (leukemia inhibitory factor) et IL6 (interleukine-6) illustre particulièrement bien comment peut être engendrée de la diversité de réponse biologique par l'utilisation de combinaisons variées d'un petit nombre de composants, dont aucun n'est spécifique d'un signal donné. Nous avons déjà vu que les récepteurs de CNTF, OSM, I.IF et II.6 partagent une sous-unité commune, gpl30, homodimérisée (IL6) ou hétérodimérisée avec une sous-unité I.IFR $\beta$ commune aux récepteurs de L.IF, OSM et CNTF ( $\mathrm{m} / \mathrm{s} n^{\circ} 5$, vol. 8 , p. 490). De plus, un déterminant supplémentaire de spécificité est indispensable en ce qui concerne IL6 (IL.6R $\alpha)$ et CNTF (CNTFR $\alpha)$ [12]. Une équipe multinationale, américaine (Tarrytown, NY et Memphis, TN), et française (Institut Pasteur de Paris), vient de démontrer que les régions cytoplasmiques de gpl30 et LIFR $\beta$, partiellement homologues, forment un complexe avec diverses tyrosine kinases de la famille Jak/Tyk [12]. I a stimulation par les diverses cytokines de la famille CNTF/IL6 induit la phosphorylation de tyrosine kinases différentes dans différentes lignées cellulaires: Jakl et Jak2 (cellules EW-1), ou bien Jak2 et un faible niveau de Jakl et Tyk2 (cellules SKMES), ou encore Jakl et Tyk2 (cellules U266).

Dans les cellules hépatomateuses différenciées en culture, IL6 est l'une des cytokines essentielles de l'activation des gènes de "la phase aiguë" (arute phase) de l'inflammation. Elle induit la phosphorylation de Jakl et Tyk2 (comme dans les cellules U266), et de deux facteurs de transcription, Stat9l (comme indiqué cidessus) et APRF (acute phase response factor), une protéine de $89 \mathrm{kDa}$ qui se fixe à l'élément d'ADN conférant la réponse transcriptionnelle à IL6 (IL6-RE). APRF est immunologiquement relié à Stat9l et IL6-RE est assez similaire à GAS [14]. L'équipe, particulièrement internationale (Aixla-Chapelle et Martinsried en Allemagne ; New York, NY, USA ; Berne, Suisse; Victoria, Australie; Kanagawa et Osaka, Japon; Paris), responsable de ce travail propose également un schéma où la tyrosine kinase Jakl est en permanence associée au récepteur gpl30; la liaison du ligand IL.6 à ce dernier entraîne, peut-être par l'intermédiaire d'une oligomérisation des sous-unités du récepteur, la phosphorylation des tyrosine kinases, principalement Jakl et, à un moindre degré, Tyk2. APRF interagit alors avec le complexe kinase-récepteur activé, et est phosphorylé sur une tyrosine, ce qui conduit à son passage dans le noyau et à l'activation des gènes de l'acute phase possédant un élément de type IL6-RE dans leur région régulatrice. D’autres cytokines entraîneraient l'oligomérisation de gpl30 avec la sous-unité I.IFR $\beta$ dont le domaine cytoplasmique peut interagir avec des kinases légèrement différentes de celles liées à̀ gpl30, ce qui peut engendrer un signal différent de celui déclenché par IL6 [14].

\section{Des cascades de phosphorylations}

La figure 1 schématise les connaissances actuelles de la transmission directe du signal entre des récep- teurs de facteurs de croissance ou de cytokines et le noyau, par l'intermédiaire de la phosphorvlation de facteurs de transmission cytoplasmiques, dirigés ainsi vers le noyau et vers leur cible d'ADN. Dans les voies les mieux caractérisées, celles passant par les récepteurs de l'interféron $\alpha$ ou $\beta$, de l'interféron $\gamma$ et des facteurs de croissance EGF et PDGF, la liaison du ligand à son récepteur aboutit à l'activation de deux tyrosine kinases: Jakl et Tyk2, dans le cas de l'interféron $\alpha$, Jakl et Jak2 dans le cas de l'interféron $\gamma$, Jakl et l'activité tyrosine kinasique propre du récepteur dans le cas de l'EGF. La coopération entre Jakl et Jak2 activés aboutit à la phosphorylation prédominante de Stat9l. Jakl semble donc être un intermédiaire indispensable (mais non suffisant) à cette phosphorylation de Stat9l sur sa tyrosine 701. En coopération avec l'activité intrinsèque tyrosine kinasique du récepteur de l'EGF, Jakl activé induit, en outre, la phosphorylation d'autres facteurs susceptibles de se fixer sur les éléments SIE $[9,11,15]$. Ces complexes ne contenant pas Stat9l semblent avoir plus d'affinités pour l'élément SIE que pour les éléments GAS. En coopération avec Tyk2 activé, Jakl phosphorylé aboutit à l'activation de Stat113, sous-unité, avec Stat91/84, du facteur IGF3. Cette phosphorylation en cascade semble impliquer, pour certaines étapes, des domaines protéiques de type SH2 (Src homologv domain 2). En effet, la mutation du domaine SH2 de Stat9l supprime sa phosphorylation en réponse à un traitement par l'interféron $\gamma$ ou par l'EGF, et conduit à la suppression de la transmission du signal de ces cytokines [3].

Les cytokines de la famille CNTF/IL6 mettent également en jeu une coopération entre plusieurs tyrosine kinases de la famille Jak/Tyk, en des combinaisons variées selon les types cellulaires [12]. Dans les cellules hépatiques, cela aboutit à la phosphorylation de Stat91 et d'un autre facteur de la même famille, APRF [14].

Les partenaires éventuels et les cibles de Jak2, stimulés lors de la liaison de l'érythropoïétine, de l'hormone de croissance ou de 
l'interleukine-3 à leurs récepteurs, ne sont pas encore connus.

En conclusion, cette amélioration de notre compréhension des mécanismes de la transmission des signaux dans la cellule est probablement un des événements les plus importants de l'année 1993 en biologie. Rappelons-nous qu'il y a deux ans à peine, rien n'était connu de la nature des signaux envoyés par des récepteurs de cytokines ou de facteurs de croissance à la cellule. Maintenant nous savons qu'au moins deux voies de signalisation peuvent être empruntées : celle passant par les petites protéines $G$ de la famille Ras, par Raf et par les MAP kinases ; et la cascade des tvrosine kinases aboutissant à la phosphorylation activatrice de facteurs de transcription sur des tyrosines. La signification de chacun de ces signaux est imprimée par une combinatoire complexe utilisant des associations variées de protéines kinases, de facteurs de transcription et d'éléments de réponse aux spécificités voisines mais non identiques

\section{RÉFÉRENCES}

I. Müller M, Briscoe J, Laxton C, Guschin D, Ziemiecki A, Silvennoinen (), Harpur AC; Barbieri (; Witthuhn B, Schindler C, Pellegrini S, Wilks AF, Ihle JN, Stark (BR, Kerrl. The protein tyrosine kinase Jakl complements defects in interferon- $\alpha / \beta$ and $-\gamma$ signal transduction. Valure $1993 ; 366$ : 129-35.

2. W'atling D), (Guschin D, Müller M, Silvennoinen (), 'W'itthuhn BA, Quelle FW', Rogers NC, Schindler C, Stark (;R, Ihle JN, Kerr IM. Complementation by the protein tvrosine kinase Jak2 of a mutant cell line defective in the interferon- $\gamma$ signal transduction pathway. Nalure $1993 ; 366: 166-70$.

3. Shuai K, Ziemiecki A, W'ilks AF, Harpur AG, Sadowski HB, Gilman MZ, Darnell JE. Polypeptide signalling to the nucleus through tyrosine phosphorylation of Jak and Stat proteins. Nature 1993; 366 : $580)-2$.

4. Silvennoinen (), Ihle JN, Schlessinger J, Levy DE. Interferon-induced nuclear signalling by Jak protein tyrosine kinases. Nalure $1993 ; 366$ : $583-5$.

5. W'itthuhn BA, Quelle FW, Silvennoinen $O$, Yi T, Tang B, Miura O, Ihle JN. Jak2 associates with the erythropoietin receptor and is tyrosine phosphorylated and activated following stimulation with ervthropoietin. Cell 1993; 74: 227-36.

$\mathrm{m} / \mathrm{s} n^{\circ} 2 \mathrm{vol}$. 10 , férier 94
6. Silvennoinen $\mathrm{O}, \quad \mathrm{W}$ 'itthuhn BA, Quelle FW, Cleveland JL, Yi, T, Ihle JN. Structure of the murine Jak2 proteintyrosine kinase and its role in interleukin 3 signal transduction. Proc Nall Acad Sci USA 1993; 90: 8429-33.

7. Argetsinger LS, (iampbell (;S, Yang X W'itthuhn BA, Silvennoinen (), Ihie JN Carter-Su C. Identification of Jak2 as a growth hormone receptor-associated tvrosine kinase. Cell; 74 : 237-44.

8. Silvennoinen O, Schindler C, Schlessinger J, Levy DE. Ras-independent growth factor signaling by transcription factor tyrosine phosphorylation Science 1993; 261: 1736-7.

9. Sadowski l-IB, Shuai K, DarnellJE, Gilman MZ. A common nuclear signal transduction pathway activated by growth factor and cytokine receptors. Science 1993 ; 261 : 1739-43.

10. Shuai K, Stark (iR, Ker IM, Darnell Jr. A single phosphotyrosine residue of Stat91 required for gene activation by interferon\%. Science 1993; 261: 1744-6.

11. Fu XY, Zhang IJ. Transcription factor P9l interacts with the epidermal growth factor receptor and mediates activation on the $c$-fos gene promoter. Cell 1993; 74 : $1135-45$.

12. Stahl N, Boulton TG; Fanuggella T, Ip NY, Davis S, Witthuhn BA, Quelle FW, Silvennoinen (), Barbieri (;, Pellegrini S, Ihle JN, Yancopoulos (;D. Association and activation of Jak-Tyk kinases by (NNTF-LIFOSM-IL-6 $\beta$ receptor components. Science 1994 ; 263 : 92-5.

13. Larner AC; David M, Feldman GM, lgarashi K. Hackett RH, W'ebb ISA, Sweitzer SM, Petricoin III EF, Finbloom ISS. Tyrosine phosphorylation of DNA binding proteins by multiple cytokines. Science $1993 ; 261: 1730-3$.

14. Lütticken (;) W'egenka UM, Yuan J, Buschamnn J, Schindler C, Ziemieck A, Harpur AC, 'Wilks AF, Yasukawa K, Taga T, Kishimoto T, Barbieri (;, Pellegrini S, Sendtner M, Heinrich PC, Horn F. Association of transcription factor APRF and protein kinase Jakl with the interleukin-6 signal transducer gp130. Science 1994; 263 : 89-92.

15. Ruff-Jaminson S, Chen K, Cohen S. Induction by EGF and interferon- $\gamma$ of tyro sine phosphorylated DNA binding proteins in mouse liver nuclei. Science 1993; 261 : 1733-6.

Axel Kahn

Directeur de l'unité de recherches en génétique et pathologie moléculaires, Inserm U 129. ICGM, 24, rue du Faubourg Saint-Jacques, 75014 Paris, France. 